\title{
Symmetric and Asymmetric Vortex-Antivortex Molecules in a Fourfold Superconducting Geometry
}

\author{
R. Geurts, M. V. Milošević, and F. M. Peeters* \\ Departement Fysica, Universiteit Antwerpen, Groenenborgerlaan 171, B-2020 Antwerpen, Belgium
}

(Received 9 June 2006; published 28 September 2006)

\begin{abstract}
In submicron superconducting squares in a homogeneous magnetic field, Ginzburg-Landau theory may admit solutions of the vortex-antivortex type, conforming to the symmetry of the sample [L. F. Chibotaru et al., Nature (London) 408, 833 (2000)]. Here we show that these fascinating, but never experimentally observed states, can be enforced by artificial fourfold pinning, with their diagnostic features enhanced by orders of magnitude. The second-order nucleation of vortex-antivortex molecules can be driven by either temperature or an applied magnetic field, with stable asymmetric vortex-antivortex equilibria found on its path.
\end{abstract}

DOI: 10.1103/PhysRevLett.97.137002

All subatomic particles have a fraternal twin: an antiparticle, exactly alike except for, e.g., opposite charge or helicity. However, as a general rule, matter and antimatter cannot coexist without excess energy and annihilating each other.

This universal duality has its analogue in the physics of superconductors, where vortices as the carriers of magnetic flux play the role of charged particles. Namely, vortexantivortex pairs in superconducting films can be induced in a local hot spot, created by thermal fluctuations [1], photon absorption [2], or driving current [3]. Intuitively, vortexantivortex pairs are easily stabilized in an inhomogeneous magnetic field, such as one resulting from dipolar magnetic objects in the vicinity of the superconductor [4].

Contrary to the latter examples, recent theoretical studies [5-7] have shown that vortex-antivortex structures can be stabilized in submicron superconductors even in a homogeneous magnetic field, and without any apparent energy input. These findings are restricted to the cases when the vortex structure does not conform with the sample geometry. A typical example is the $C_{3}$ symmetry of the three-vortex state in a superconducting square; in spite of the unipolar applied field, the $C_{4}$ state of four vortices with a central antivortex may become energetically preferable. Similarly to the solid-fluid and the ferromagnetic symmetry-breaking processes, the more symmetrical phase is found on the high-temperature side of the transition, and the less symmetrical one on the low-temperature side. Landau pointed out that one can always unequivocally determine whether given state possesses a given symmetry [8]. Therefore, it is not possible to analytically deform a state in one phase into a phase possessing truly different symmetry. This means, for example, that it is impossible for the solid-liquid phase boundary to end in a critical point like the liquid-gas boundary. Nevertheless, the above described vortex-antivortex nucleation during the $C_{3}$ to $C_{4}$ transition in mesoscopic superconductors is of second order with respect to temperature. The appearance and stability of these asymmetric vortex-antivortex
PACS numbers: 74.20.De, 45.05.+x, 74.25.Dw, 74.78.Na

molecules in the ground state is one of the main objectives of this Letter.

It should be noted here that the symmetry-induced vortex-antivortex configurations remain experimentally undetected, mainly because of their high sensitivity to defects in sample edges [6]. As another drawback, due to extreme vortex proximity [7], those states are undistinguishable from a single multiquanta vortex for conventional techniques such as scanning-tunneling and Hallprobe microscopy. Theory suggests that the latter problem can be solved by a magnetic dot deposited on the sample [9]. An added inhomogeneous magnetic potential interacts dually with the present vortex and antivortex [10] and separates them in a controllable fashion, but disturbs the conceptual novelty of no external stabilizing factor added to the vortex-antivortex coexistence.

To address above issues, we discuss in this Letter the properties and the improved observation conditions of a vortex-antivortex state in a square superconducting sample with fourfold pinning. We introduce a cluster of $2 \times 2$ nanoholes to enforce the $C_{4}$ symmetry, the nonenergetic source of vortex-antivortex nucleation (see Fig. 1). Albeit, induced states do not always obey the imposed symmetry. We show that similar symmetry breaking can be achieved in a classical cluster of oppositely charged particles in a strongly confined geometry. The latter setup has recently emerged as the "standard model" for a variety of systems

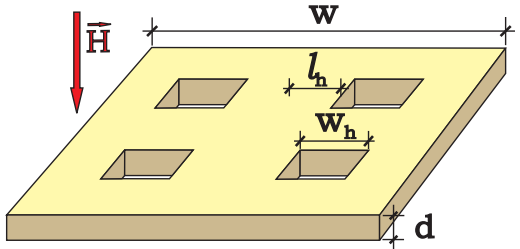

FIG. 1 (color online). Superconducting square (size $w \times w$ and thickness $d$ ) with four nanoholes (each $w_{h} \times w_{h}$ in size, displaced by $l_{h}$ from $x$ and $y$ symmetry lines). Indicated direction of applied homogeneous magnetic field $H$ is denoted as positive. 
on different energy and length scales, not only flux lines in superconductors, but also vortices in Bose-Einstein condensates, electrons on liquid helium, colloidal suspensions, and dust particles in plasmas.

Our theoretical approach relies upon the GinzburgLandau (GL) theory, where we solve self-consistently a set of mean field differential equations for the order parameter $\psi$ and the vector potential $\mathbf{A}$ :

$$
\begin{gathered}
(-i \nabla-\mathbf{A})^{2} \psi=\left(1-T-|\psi|^{2}\right) \psi, \\
-\kappa^{2} \nabla \times \nabla \times \mathbf{A}=\mathbf{j} .
\end{gathered}
$$

The latter is the Maxwell-Ampère equation with a current density $\mathbf{j}=\operatorname{Im}\left(\psi^{*} \nabla \psi\right)-|\psi|^{2} \mathbf{A} . \boldsymbol{\kappa}$ is the material property and equals the ratio between the magnetic field penetration depth $\lambda$ and coherence length $\xi$. The solution of Eqs. (1) and (2) minimizes Gibbs free energy $G / G_{0}=$ $\Omega^{-1} \int\left[-|\psi|^{4}+2\left(\mathbf{A}-\mathbf{A}_{0}\right) \cdot \mathbf{j}\right] d \Omega$, where the condition $j_{\perp}=0$ was used on the boundaries of the superconducting volume $\Omega$, and $G_{0}$ stands for the superconducting condensation energy $H_{c}^{2} / 4 \pi$. In above expressions, $\nabla \times \mathbf{A}_{0}=\mathbf{H}$ denotes the applied magnetic field, and $\nabla \times \mathbf{A}=\mathbf{h}$ the resulting local field in the sample. All distances are expressed in units of $\xi_{0}=\xi(T=0)$, the vector potential in $\phi_{0} / 2 \pi \xi_{0}$, and the order parameter in $\sqrt{-\alpha / \beta}$ with $\alpha, \beta$ being the GL coefficients. For details of the numerics, we refer to Ref. [11].

Symmetry-induced asymmetry.-Figure 2 shows the ground-state phase diagram of our perforated sample, compared to the previously studied one without holes [5$7,9,12]$. Namely, at given temperature $T$ (scaled to critical temperature $T_{c}$ ), we obtain stable solutions of Eqs. (1) and (2) by starting the iterative procedure from randomly generated initial conditions, while applied magnetic field $H$ is

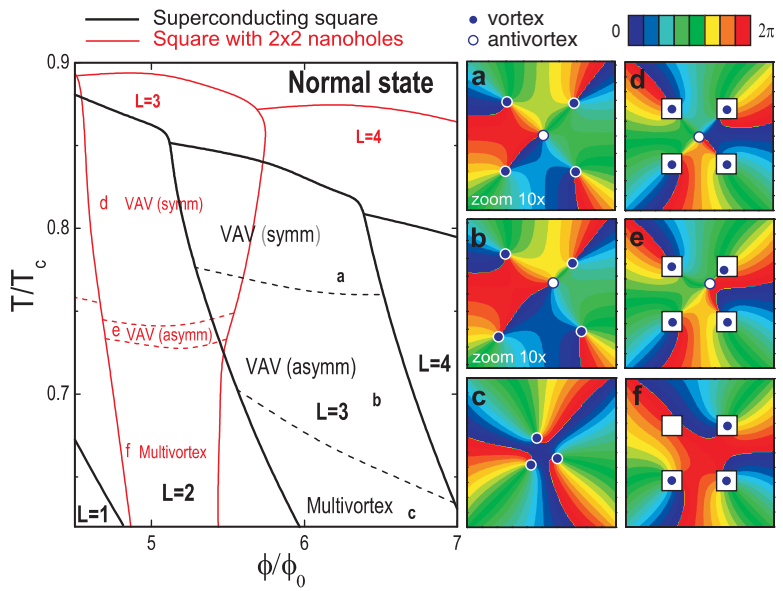

FIG. 2 (color online). Temperature versus magnetic field equilibrium phase diagram. Solid (dashed) lines illustrate first (second) order transitions in the ground state. Parameters used: $\kappa=1, d=1 \xi_{0}, w=10 \xi_{0}, l_{h} / w=w_{h} / w=0.125$ (see Fig. 1). Contour plots (a)-(f) show the phase of the order parameter of particular vortex states (as indicated in the left diagram). swept up or down. The ground state is then determined by comparing the energy of all found states. Note that dimensions of the system are given in $\xi_{0}$, so that depending on the material our results apply to samples from hundreds of nanometers to several microns in size. Parameter $\kappa$ is taken equal 1.

The size-field phase diagram [size scaled to $\xi(T)=$ $\xi_{0} / \sqrt{1-\left(T / T_{c}\right)^{2}}$ ] for a square sample without holes has been studied previously in Refs. [5,7]. Since in Ref. [5] the linearized GL theory is used, valid only extremely close to the superconducting/normal phase boundary, those results were quantitatively corrected in Ref. [7] where both GL equations were numerically solved. However, authors kept parameter $\kappa_{\text {eff }}=\kappa^{2} \xi(T) / d$ fixed when the temperature was changed, so their diagram did not reflect the true temperature dependence of the vortex state. Nevertheless, our phase diagram (with temperature dependence taken explicitly) shows similar qualitative behavior-sets of individual vortices are found at lower temperatures, which merge into a giant vortex at higher temperatures for all vorticities $(L)$ except $L=3$. The triangular symmetry of that state [Fig. 2(c)] is not favorable closer to $T_{c}$ when square confinement dominates, and the $L=4-1(=3)$ vortex-antivortex state is induced [Fig. 2(a)].

However, contrary to previous works, our analysis shows that the vortex-antivortex (VAV) nucleation process is of second order (dashed lines in Fig. 2) over a broad temperature and field range. In other words, with changing applied magnetic field $\left(\Delta \phi \approx \phi_{0}\right)$ or temperature $(\Delta T \approx$ $0.1 T_{c}$ ) while in the $L=3$ state, besides the three existing vortices a vortex-antivortex pair gradually dissociates. A remarkable nonsymmetric $L=4-1$ state is induced [Fig. 2(b)], and the ground-state exhibits symmetry breaking during the transition between intrinsically symmetric states $\left(C_{3}\right.$ vs $\left.C_{4}\right)$.

Although these asymmetric equilibria seem fairly counterintuitive, their stability follows from the competing interactions in this system. In analogy, we recall the properties of small confined clusters of particles, where the confinement would result from the screening currents in our system (along the sample edges), and charged particles represent vortices.

Analogy to classical systems. - The model system was defined in Ref. [13], where the Hamiltonian is given by $\mathcal{H}=\sum_{i>j}^{N} U_{i j}+\sum_{i}^{N} V(x, y)$, with $N$ being the number of particles. We took square-parabolic confinement potential of size $w_{c}$, i.e., $V(x, y)=\frac{1}{2} m \omega_{0}^{2} \frac{x^{2}+y^{2}}{w_{c}^{2}}[1+$ $\left.\sqrt{\delta+\cos ^{2}\left(2 \arctan \frac{y}{x}\right)}\right]$ ( $m$-mass of particle, $\omega_{0}$-confinement frequency) with a nonzero $\delta \ll 1$, ensuring the existence of the derivatives of this potential in the corners of the square. To further translate this system to vortices in mesoscopic superconductors, one has to choose properly the vortex-(anti)vortex interaction energy $\left(U_{i j}\right)$. First, this interaction must be of long range (and diminishing far from the source), knowing that supercurrents decay $\sim 1 / r$ away 
from the vortex. Second, $U_{i j}$ may not diverge for $r_{i j} \leq \alpha$ but saturate, allowing for realistic merging of vortices into a giant vortex [14] or a vortex-antivortex annihilation. In this scenario, $\alpha$ roughly corresponds to the finite size of the vortex core.

Although any interaction energy of described profile would suit our analysis, we used a modified logarithmic and modified Coulomb interaction, more specifically, $d U / d r\left(r_{i j}\right)=\beta\left(1-e^{-r_{i j} / \alpha}\right)^{2} / r_{i j} \quad$ and $\quad d U / d r\left(r_{i j}\right)=$ $\frac{q_{i} q_{j}}{\varepsilon}\left(1-e^{-r_{i j} / \alpha}\right)^{3} / r_{i j}^{2}$, respectively. Notice that the modified Coulomb interaction is a quality fit for $\alpha^{-1}=\sqrt{2} \kappa$ (up to a multiplying constant) to the realistic vortex-vortex interaction in type-II samples (see, e.g., Ref. [15]).

Next we employed molecular dynamics simulation, a relatively simple and rapidly convergent technique that gives a reliable estimate of the energy for small clusters of interacting particles. The obtained energy versus confinement size $\left(w_{c}\right)$ is shown in Fig. 3, for a cluster containing three particles of the same charge (i.e., $U_{i j}>0$ ) and a cluster of four particles and an antiparticle, with clear analogy to our $L=3$ and $L=4-1$ vortex state. For large $w_{c}$, the ground states of the two clusters have identical energy, as one particle and the antiparticle sit on top of each other. However, for tighter confinement, the $N=$ $4^{+} 1^{-}$cluster attains lower energy than the $N=3^{+}$one. Namely, particle and antiparticle gradually separate causing the rearrangement of the remaining 3 particles. Figures 3(a)-3(c) show snapshots of this dynamical transition in the ground state. To our knowledge, this symmetry breaking in the crystallization of confined systems is novel and applies to classical particles interacting with charged impurities in the substrate [16], and potentially to small ionic crystals under pressure [17]. Note also that colloidal molecules have recently been realized experimentally on hydrophilic square templates [18].

Distinction of antivortex in fourfold pinning. -As shown in Fig. 2, in our sample with nanoholes, the sym-

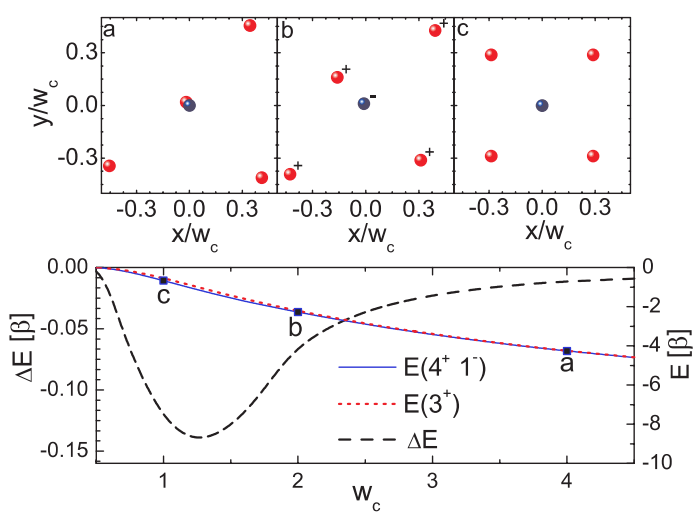

FIG. 3 (color online). Energy of the 3-particle cluster compared to the cluster of 4 particles and 1 antiparticle, as a function of the size of the square confinement $w_{c}$, for modified logarithmic interparticle interaction (of strength $\beta$, and with $\alpha=1$ ). (a) -(c) are snapshots of the ground-state configuration of the latter cluster with decreasing $w_{c}\left(x, y\right.$ in units of $\left.\sqrt{\frac{2 \beta}{m \omega_{0}^{2}}}\right)$. metrically placed perforations (i) pin (attract) vortices individually, i.e., favor the multivortex state, and (ii) stabilize vortex states commensurate with the square geometry, e.g., $L=2$ and particularly $L=4$ [19]. Although the $L=3$ state is somewhat suppressed in the $H-T$ space compared to the plain square case, the antivortex in the $L=4-1$ state is clearly pronounced [see Figs. 2(d) and 2(e)] since the remaining 4 vortices are captured by the holes. To better illustrate this issue, we calculated the vortex-antivortex distance $\left(d_{\mathrm{VAV}}\right)$ and amplitudes of the Cooper-pair density and local magnetic field between them, as a function of the sample parameters. As shown in Fig. 4, we are able to achieve the vortexantivortex distance of $5 \%-20 \%$ of the sample size, which greatly exceeds the distances found in plain squares (see Ref. [7]), especially in larger samples. At the same time, the amplitudes of both the order parameter and the local magnetic field are enhanced by almost 2 orders of magnitude compared to previous studies, and reach the limits of conventional scanning techniques. Our study shows that $w_{h}=l_{h}=12.5 \% \mathrm{w}$ are optimal parameters for the observation of the antivortex, as larger holes capture more applied flux and decrease the field in the center of the sample, whereas larger distance between the holes results in a weaker influence on the vortex-antivortex state in the
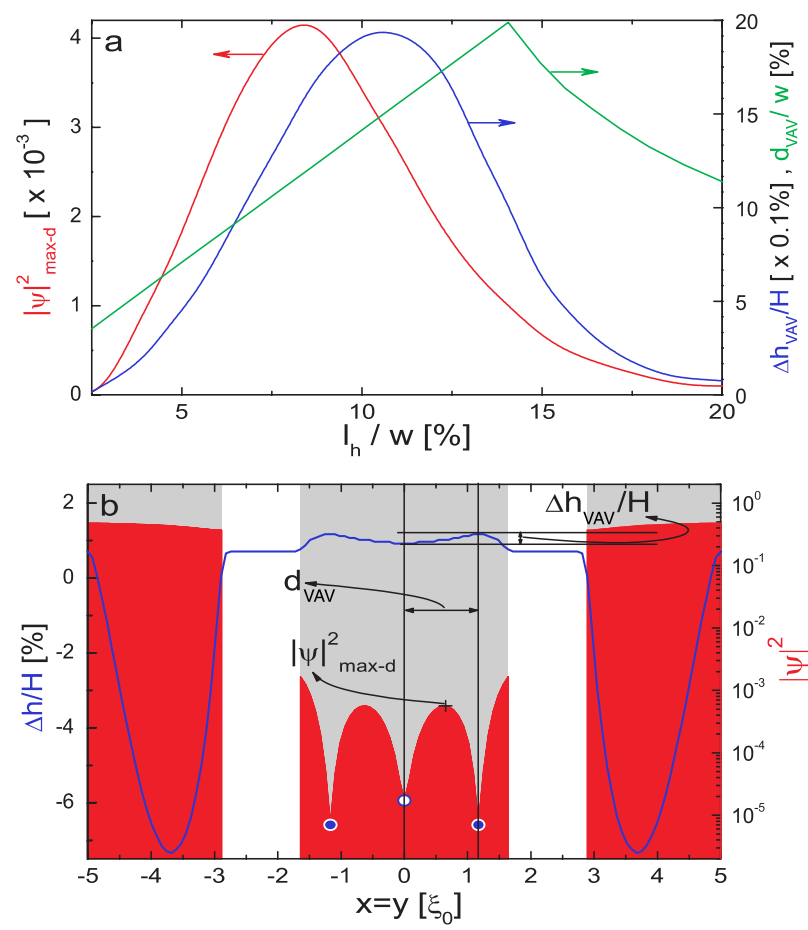

FIG. 4 (color online). The properties of the $L=4-1$ vortexantivortex state in the perforated sample, at $T=0.84 T_{c}$, and applied flux $\phi=5.5 \phi_{0}$ : (a) the distance, maximal $|\Psi|^{2}$ density, and difference in the magnetic response between vortex and antivortex, as a function of the position of the holes; (b) the Cooper-pair density and the magnetic field profile $(\Delta h=h-$ $H$ ) along the diagonal of the sample, in the case when vortices are not residing in holes $\left[l_{h}=0.165 \mathrm{w}\right.$, see (a)]. 


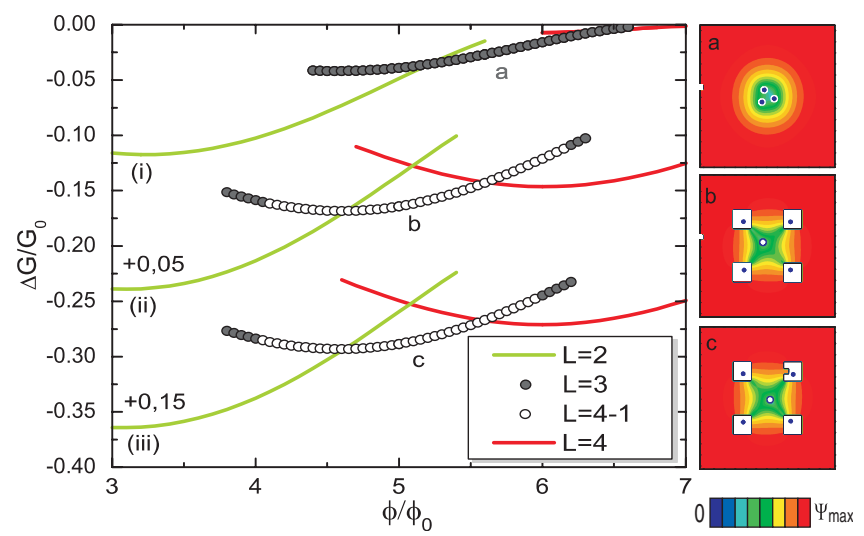

FIG. 5 (color online). Gibbs free energy for $T=0.78 T_{c}$ and (i) plain square sample with an edge defect (size $0.5 \xi_{0}$ ), (ii) sample with $2 \times 2$ holes and an edge defect, and (iii) sample with $2 \times 2$ holes and a defect in hole edges. Insets (a)-(c) show the Cooper-pair density plots of found states with total vorticity 3 , in cases (i)-(iii).

sample center (see Fig. 4). It should also be emphasized that fourfold distribution of holes is more important than the actual shape of the sample; i.e., even a circular disk with four symmetrically arranged holes exhibits the $L=$ 4 - 1 state.

One should note that the intervortex interaction is governed by $\kappa$, and may even change sign in type-I samples [15]. This strongly affects the vortex configurations, and consequently the vortex-antivortex stabilization. In our calculation for both squares with and without nanoholes, a decrease of $\kappa$ did not favor the $L=4-1$ state but instead the $L=3$ giant vortex becomes more stable, contrary to the findings of Ref. [12]. Apparently, suggested vortex-antivortex repulsion at lower $\kappa$ is dominated by attraction between the remaining vortices. On the other hand, for larger $\kappa$, the shorter range of intervortex interactions reduces the stability of asymmetric states which rely on strong competing forces. Therefore, material properties are very important for potential experimental observation of the antivortex, as $\kappa$ can be arguably controlled by impurities, which increase $\lambda$ and decrease $\xi$.

Enhanced stamina of vortex-antivortex states. - Yet another crucial experimental issue is the sensitiveness of the vortex-antivortex state to surface defects. While the ground state strongly depends on symmetry, the analysis of Ref. [6] has shown that defects as small as $0.01 w$ at the edges of a plain superconducting square disable vortexantivortex nucleation. We found that $2 \times 2$ nanoholes significantly strengthen the $L=4-1$ state. Figure 5 shows the free energy diagrams of (i) a plain square with a small edge defect, (ii) a square with nanoholes and an edge defect, and (iii) square with nanoholes and a defect at one of the holes. Whereas in case (i) no vortex-antivortex state is found, in both cases (ii) and (iii) we found stable $L=4-1$ state. Our numerical experiment shows that vortex-antivortex states in latter cases can survive for defects in edges up to $10 \%$ of the sample size and defects in nanoholes up to a remarkable $40 \%$ of their size. Note also that the presence of defects changes the current profile of the sample, which again results in asymmetric vortexantivortex configurations [see Figs. 5(b) and 5(c)].

Therefore, we may conclude that artificial fourfold pinning vigorously enhances (in all relevant aspects) the experimental observability of vortex-antivortex molecules in flat superconducting samples. The verification of the found asymmetric vortex-antivortex equilibria is of particular importance, as this symmetry breaking is predicted both for many-body and few-body systems with strong competing interactions in polygonal constraints.

This work was supported by the Flemish Science Foundation (FWO-Vl), the Belgian Science Policy, the JSPS/ESF-NES program, and the ESF-AQDJJ network.

*Electronic address: francois.peeters@ua.ac.be

[1] V. L. Berezinskii, Zh. Eksp. Teor. Fiz. 59, 907 (1970); J. M. Kosterlitz and D. J. Thouless, J. Phys. C 6, 1181 (1973); M. R. Beasley et al., Phys. Rev. Lett. 42, 1165 (1979).

[2] A. M. Kadin et al., Phys. Rev. Lett. 65, 3193 (1990).

[3] K. Epstein et al., Phys. Rev. Lett. 47, 534 (1981); M. V. Milošević et al., Phys. Rev. Lett. 95, 147004 (2005).

[4] M. J. Van Bael et al., Phys. Rev. Lett. 86, 155 (2001); M. Lange et al., Phys. Rev. Lett. 90, 197006 (2003); Phys. Rev. B 72, 052507 (2005); M. V. Milošević and F. M. Peeters, Phys. Rev. Lett. 93, 267006 (2004); 94, 227001 (2005).

[5] L. F. Chibotaru et al., Nature (London) 408, 833 (2000); Phys. Rev. Lett. 86, 1323 (2001); Phys. Rev. B 70, 094505 (2004).

[6] A. S. Mel'nikov et al., Phys. Rev. B 65, 140503 (2002).

[7] J. Bonča and V. V. Kabanov, Phys. Rev. B 65, 012509 (2002); B. J. Baelus and F. M. Peeters, Phys. Rev. B 65, 104515 (2002); T. Mertelj and V. V. Kabanov, Phys. Rev. B 67, 134527 (2003).

[8] P.W. Anderson, Basic Notions of Condensed Matter Physics (Westview Press, Oxford, 1984).

[9] C. Carballeira et al., Phys. Rev. Lett. 95, 237003 (2005).

[10] M. V. Milošević and F. M. Peeters, Phys. Rev. B 68, 094510 (2003).

[11] V.A. Schweigert and F. M. Peeters, Phys. Rev. B 57, 13817 (1998)

[12] V. R. Misko et al., Phys. Rev. Lett. 90, 147003 (2003).

[13] V. M. Bedanov and F. M. Peeters, Phys. Rev. B 49, 2667 (1994).

[14] A. Kanda et al., Phys. Rev. Lett. 93, 257002 (2004).

[15] E. H. Brandt, J. Low Temp. Phys. 53, 41 (1983).

[16] G. A. Farias et al., Solid State Commun. 100, 711 (1996).

[17] P. T. Jochym et al., Phys. Rev. B 65, 024106 (2001).

[18] N. A. Bernotski et al., "Formation of Two-Dimensional Colloidal Sphere Arrays on Micro-Patterns," in Proceedings of the 2nd Annual Conference on Foundations of Nanoscience: Self-Assembled Architectures and Devices (FNANO), Snowbird, UT, USA, 2005, http://science technica.com/Foundations-of-Nanoscience-2005.cfm.

[19] G. R. Berdiyorov et al., Phys. Rev. B 68, 174521 (2003). 